\title{
Cyclic Ion Mobility - Collision Activation Experiments Elucidate Protein Behaviour in the Gas-Phase
}

Charles Eldrid[a], Jakub Ujma[b], Hannah Britt[a], Tristan Cragnolini[c], Symeon Kalfas[a], Dale CooperShepherd[b], Nick Tomczyk[b], Kevin Giles[b], Mike Morris[b], Rehana Akter[d], Daniel Raleigh[a,d] and Konstantinos Thalassinos*[a,c]

\author{
[a] Institute of Structural and Molecular Biology \\ Division of Bioscience, University College London, London, WC1E 6BT, UK \\ E-mail:k.thalassinos@ucl.ac.uk \\ [b] Waters Corporation, Wilmslow, SK9 4AX, UK \\ [c] Institute of Structural and Molecular Biology, \\ Birkbeck College, University of London, London, WC1E 7HX
}

[d] Stony Brook University, 100 Nicolls Rd, NY 117794, USA

Supporting information for this article is given via a link at the end of the document.

\begin{abstract}
Elucidating the properties of intrinsically disordered proteins (IDPs) and unfolded and partially folded states of globular proteins is challenging owing to their heterogeneous and dynamic nature. Protein unfolding and misfolding is a key feature of a broad range of debilitating diseases, whilst the conformational propensities of intrinsically disordered proteins can play a significant role in modulating their activity, and the properties of unfolded states of globular proteins modulates their stability and tendency to aggregate. Ion mobility-mass spectrometry (IM-MS) is a powerful method for interrogating these systems, however limits in resolution and the difficulty in probing the energetics of interconversions amongst heterogeneous ensembles are major issues. Herein, using a quadrupole/cyclic-IM/ time-of-flight MS instrument, we show how the combination of precursor mass selection, mobility selection (IMn) and collisional activation (CA) allows the elucidation of complicated gasphase dynamic behavior. The methodology employed is general and is demonstrated using a classic model globular protein, cytochrome $\mathrm{C}$, and an aggregation-prone IDP, amylin. CA allows investigations of protein conformational dynamics and unfolding in the gas-phase for heterogeneous mixtures, whilst the additional precursor mass selection capability provides high resolution and selectivity, facilitating more in-depth investigation. Understanding protein dynamics in the gas-phase will allow greater insight into protein behaviour and allow application of gas-phase techniques to clinically relevant systems.
\end{abstract}

The conformational properties of the unfolded state of proteins can influence their stability, their tendency to aggregate and even their function. Closely related to the problem of characterizing protein unfolded states is the quest to develop a detailed description of intrinsically disordered proteins (IDPs). IDPs form a significant fraction of the proteome and while such proteins often adopt structure upon binding to their partners, the properties of their free state can influence their function. Protein unfolding and misfolding also play a central role in a broad range of debilitating disorders with notable examples including prion diseases, Alzheimer's, Parkinson's and type II diabetes, amongst others.[1-4] Classical structural biology techniques such as $\mathrm{x}$-ray crystallography, cryoelectron microscopy and nuclear magnetic resonance struggle to capture either the dynamics or heterogeneity of such systems and often require significant amounts of material. Ion mobility coupled to mass-spectrometry (IM-MS) allows a measurement of 3-dimensional structure, in the form of collision cross-section (CCS), as well as rapid identification of varieties of oligomeric states from small quantities of heterogenous samples.[5-9] However, limits in resolution and the difficulty in probing the energetics of interconversions amongst heterogeneous ensembles are major issues.

IM-MS can provide useful information on protein dynamics, and has been used extensively to study gas-phase structures of proteins;[10-12] however, a greater depth of information can be gained by activating ions prior to, or during, the first stages of IM analysis. These methods are usually referred to as either collision induced unfolding (CIU), injection activation, or collision activation (CA).[10,13-16] By measuring the unfolding of 
proteins in the gas-phase it is possible to understand important features such as the preferential binding of different sub-units within a complex, the stabilisation of conformations upon drug binding, the number of domains present in a protein, and antibody arm swapping.[16-20]

Multi-stage IM selection/ separation (IMn) and activation allows direct probing of conformational subpopulations and gives greater insights into unfolding pathways and the behaviour of specific states.[21,22] Until recently, multi-stage IM instruments were custom-built in research laboratories, however a new generation of instruments capable of multiple IM selections are becoming available and offer the possibility of studying heterogeneous protein ensemble under native conditions.[23,24]

Herein we employ IMn and collisional activation, using a multi-pass cyclic IM (cIM) instrument,[23,24] which we refer to as slice-CA, and show how it can be used to understand in much greater detail gas-phase protein unfolding and conformational interconversions. We demonstrate the utility of the method using a commonly studied globular protein, equine cytochrome $\mathrm{C}(\mathrm{CytC})$, as well as human islet amyloid polypeptide (hIAPP), also referred to as amylin, which is a 37 residue intrinsically disordered polypeptide implicated in the pathogenesis of type II diabetes.

The +7 charge state of CytC was quadrupole isolated and injected with varying levels of activation energy into the cIM. Varying injection activation produced three conformational profiles: a native profile $(+0 \mathrm{~V})$, activated intermediate states $(+10 \mathrm{~V})$, and highly activated extended states $(+45 \mathrm{~V})$. After one pass around the cIM, arrival time distribution (ATD) slices of $4 \mathrm{~ms}$ (Figure 1A, E and I, green, yellow and red slices respectively for native, intermediate and extended) were extracted to a store, then reinjected with further activation for additional IM separation.

The native ATD (Figure 1A, population a) presents as a single feature with an extended tail. This population of ions has been previously subjected to multiple passes around the cIM however we were not able to resolve them into more distinct features.[23] Slice-CA overcomes this limitation and reveals structural transition from a to a compact intermediate $\beta$, which further transitions into multiple distinguishable intermediate conformational states $(\gamma-\varepsilon$, figure $2 A)$. The activated intermediate population presents as a variety of features, some of which could be seen previously $(\alpha-\varepsilon)$, and a new low intensity population $\zeta$ (Figure $1 \mathrm{H}$ ). Activation of these populations leads to direct conformational transitions from $a-\varepsilon$ and then $\varepsilon$ to $\zeta$ and $\eta$ (Figure 2B). The intensity of population $\eta$ is low in comparison to the others, and so is more easily visible in other plots (Figures S5).

Activation of the extended state shows some similarities to that of the intermediate, however, we are able to probe population $\eta$. Without additional activation energy, selection of $\eta$ (Figure $1 \mathrm{~L}$ ) produces a mixture of $\varepsilon, \zeta$ and $\eta$, suggesting that interconversion from $\eta$ is occurring spontaneously or that some minimal activation is occurring during the trapping and separation processes, which allows $\eta$ to revert back to $\varepsilon$ and $\zeta$. This is further evidenced by peak asymmetry resulting in a "bridge" between the peaks of $\varepsilon$, $\zeta$ and that of $\eta$ (Figures S4-7). Under activation, the intensity of $\eta$ drops with a corresponding rise in intensity of $\varepsilon$ and $\zeta$. It appears that $\varepsilon$ and $\zeta$ are highly stable kinetically trapped states, as evidenced by the very high energy required to induce a conversion from $\varepsilon$ to $\zeta$ (Figure $2 C$ ). Interestingly, $\zeta$ produced from $\eta$ appears as a sharper peak than is initially present as $\zeta$ (Figures 2C, S5-6). This may indicate other interconversion processes occurring in the conformational ensemble observed initially (causing peak asymmetry, broadening and "bridges", Figure S7) or the presence of an unresolved intermediate (Figure 1I, 2C). We anticipate this will be the focus of a future study.

Having demonstrated the power of the approach to studies of globular protein unfolding we next examined an IDP. hIAPP is a polypeptide hormone which is co-secreted with insulin. It normally plays an adaptive role in metabolism and affects satiation, gastric emptying, energy homeostasis and glucagon secretion.[1] However the polypeptide aggregates to form amyloid deposits in the pancreas, a process which is toxic to the insulin producing Lx-cells. hIAPP forms amyloid fibrils over the course of hours, the intermediate stages of which exhibit cellular toxicity, that is involved in type II diabetes. There is considerable interest in understanding how the structure and dynamics of hIAPP are related to toxicity and amyloidogenicity. Such information is relevant 
for developing new treatments for type II diabetes and for creating soluble IAPP analogs for use as adjuncts to insulin therapy.[25-27]

The mass spectrum of hIAPP showed a mixture of low order oligomers with overlapping charge state distributions (Figure S8). Two mobility species were observed at $\mathrm{m} / \mathrm{z} 1952$ and, following quadrupole isolation, were determined by isotopic peak spacing (Figure $3 \mathrm{~A}-\mathrm{C}$ ) to be a mixture of +2 monomer and +4 dimer. An early slice and a late slice were taken from the dimeric species (Figure 3A, blue and red respectively) for slice-CA. Under activation, the two distinct species merge to occupy the same conformational space, before eventually dissociating to give monomer at high activation voltages (Figure 3D-F). This conformational interconversion may be indicative of a low energy barrier between the two states and has not been previously observed.

The experiments enabled by the Q-cIM-ToF instrument described here reveal new insights into the conformational behavior of proteins in the gas phase. Application of the methodology to the native state of CytC allowed identification of lowly populated conformers that until now have proved unresolvable. Furthermore, use of the technique to study native and intermediate states allowed mapping of the unfolding pathway of CytC through both direct and sequential series of conversions. Application to the extended state lead to observation of states beyond the selected sub-populations, suggestive of either low energetic barriers between these excited states requiring only minimal activation, or interconversion between gas-phase conformations taking place on time-scales where stable gas-phase conformations would be expected. This behaviour has been seen a number of times in the gas-phase: the stable interconversion of activated states after relaxation or cooling.[28-30] It is tempting to relate these structures to solution refolded states, however without CCS measurements and accompanying molecular dynamics simulations these states cannot be directly related to a structural model.[31] we expect that the increased information content of the approach demonstrated here will help to motivate such studies.

The slice-CA measurements on the dimer of the amyloidogenic IDP hIAPP revealed distinct gas-phase protein behaviour with conformational interconversion evident upon activation (prior to dissociation); this is not observable with conventional single stage IM experiments, is suggestive of a low energy barrier between the states. While hIAPP has been studied using conventional IM-MS, a recent study found that differences in CCS, CCS distributions and relative population intensities could not be related to variations in cellular toxicity or amyloidogencity of hIAPP analogues.[32] The interconversion observed here opens a new avenue of study for dissecting the relationship between sequence, structure and dynamics and the toxicity and amyloidogencity of hIAPP.

The results reported here demonstrate that the slice-CA methodology is able to distinguish between different types of protein gas-phase behavior, and allows rapid identification of interconverting species, and will therefore be of great importance in studies of protein dynamics and misfolding. We note that while the terminology "CIU" is commonly used in the field, our data show that unfolding does not always occur during activation. A similar observation has been made recently with the small peptide substance P.[22] While substance $P$ is much smaller than CytC or hIAPP, it highlights that this interconversion behaviour could be widespread. However, these phenomena have not yet been studied extensively due to limitations in the currently available methods. The approach demonstrated here overcomes these limitations. IM2 was used in this work, however, the geometry of the Q-cIM-ToF instrument allows multiple mobility selections to be performed which could reveal even finer details regarding the behavior of proteins during activation conditions in the gas phase.

\section{Experimental Section}

Equine CytC (Merck Millipore, UK) was sprayed in $200 \mathrm{mM}$ ammonium acetate, at $10 \mu \mathrm{M}$, hIAPP was sprayed from $100 \mathrm{mM} \mathrm{pH} 7.4$ ammonium acetate, $1 \%$ DMSO, at a concentration of $32 \mu \mathrm{M}$, after 100X dilution from 100 $\%$ DMSO. hIAPP was synthesized using Fmoc solid phase synthesis and purified ia HPLC as previously described (supporting methods).[33] Capillaries were produced in-house using a Flaming-Brown P97 micropipette puller and coated using a Quorum Q150RS sputter coater. The prototype cIM-MS instrument (Waters Corporation, UK) and modes of acquisition are described in detail elsewhere.[23,24] Detailed acquisition parameters are 
described in the supplementary. Data were analysed using Masslynx v4.1 (Waters Corporation), Driftscope v2.1 (Waters Corporation), and in-house software which allows multi-dimensional alignment and selection of the cyclic data [Cragnolini, T. et al., manuscript in preparation]. CA fingerprint plots were created using Benthesikyme.[34] Population tracking was performed as described previously (Figures S2-4).[23]

\section{Acknowledgements}

C.E. is funded by a BBSRC iCASE Award with Waters BB/ L015382/1. H.B. and T.C. are funded by a Wellcome Trust Collaborative Awards in Science 209250/Z/17/Z. This work was also supported by Wellcome Trust award 107927/Z/15/Z J.U., N.T., D.CS., K.G., and M.M. are all employees of Waters Corporation, which manufactures and sells T-wave IM-MS instruments. We thank Dr Thomas Menneteau for producing the graphical abstract on IMn and Aisha Ben-Younis for constructive discussions.

[1] R. Akter, P. Cao, H. Noor, Z. Ridgway, L. H. Tu, H. Wang, A. G. Wong, X. Zhang, A. Abedini, A. M. Schmidt, et al., J. Diabetes Res. 2016, 2016, 1-18.

[2] I. Haq, J. A. Irving, A. D. Saleh, L. Dron, G. L. Regan-Mochrie, N. Motamedi-Shad, J. R. Hurst, B. Gooptu, D. A. Lomas, Am. J. Respir. Cell Mol. Biol. 2016, 54, 71-80.

[3] J. Gandhi, A. C. Antonelli, A. Afridi, S. Vatsia, G. Joshi, V. Romanov, I. V. J. Murray, S. A. Khan, Rev. Neurosci. 2019, 30, 339-358.

[4] F. Chiti, C. M. Dobson, Annu. Rev. Biochem. 2017, 86, 27-68.

[5] Y. Zhong, S.J. Hyung, B. T. Ruotolo, Expert Rev. Proteomics 2012, 9, 47-58.

[6] E. G. Marklund, J. L. Benesch, Curr. Opin. Struct. Biol. 2019, 54, 50-58.

[7] S. L. Bernstein, N. F. Dupuis, N. D. Lazo, T. Wyttenbach, M. M. Condron, G. Bitan, D. B. Teplow, J. E. Shea, B. T. Ruotolo, C. V. Robinson, et al., Nat. Chem. 2009, 1, 326-331.

[8] N. F. Dupuis, C. Wu, J. E. Shea, M. T. Bowers, J. Am. Chem. Soc. 2009, 131, 18283-18292.

[9] L. M. Young, P. Cao, D. P. Raleigh, A. E. Ashcroft, S. E. Radford, J. Am. Chem. Soc. 2014, 136, 660-670.

[10] K. B. Shelimov, D. E. Clemmer, R. R. Hudgins, M. F. Jarrold, J. Am. Chem. Soc. 1997, 119, 2240-2248.

[11] M. Wojnowska, J. Yan, G. N. Sivalingam, A. Cryar, J. Gor, K. Thalassinos, S. Djordjevic, Chem. Biol. 2013, 20, 1411-1420.

[12] K. J. Pacholarz, P. E. Barran, Anal. Chem. 2015, 87, 6271-6279.

[13] S. J. Valentine, D. E. Clemmer, J. Am. Chem. Soc. 1997, 119, 3558-3566.

[14] K. B. Shelimov, M. F. Jarrold, J. Am. Chem. Soc. 1997, 119, 2987-2994.

[15] S. J. Valentine, J. G. Anderson, A. D. Ellington, D. E. Clemmer, J. Phys. Chem. B 1997, 101, 3891-3900.

[16] M. P. Nyon, T. Prentice, J. Day, J. Kirkpatrick, G. N. Sivalingam, G. Levy, I. Haq, J. A. Irving, D. A. Lomas, J. Christodoulou, et al., Protein Sci. 2015, 24, 1301-1312.

[17] Y. Zhong, L. Han, B. T. Ruotolo, Angew. Chemie - Int. Ed. 2014, 53, 9209-9212.

[18] J. L. P. Benesch, J. Am. Soc. Mass Spectrom. 2009, 20, 341-348.

[19] S. M. Dixit, D. A. Polasky, B. T. Ruotolo, Curr. Opin. Chem. Biol. 2018, 42, 93-100.

[20] O. Hernandez-Alba, E. Wagner-Rousset, A. Beck, S. Cianférani, Anal. Chem. 2018, 90, 8865-8872.

[21] S. L. Koeniger, D. E. Clemmer, J. Am. Soc. Mass Spectrom. 2007, 18, 322-331. 
[22] C. R. Conant, D. R. Fuller, Z. Zhang, D. W. Woodall, D. H. Russell, D. E. Clemmer, J. Am. Soc. Mass Spectrom. 2019, 30, 932-945.

[23] C. Eldrid, J. Ujma, S. Kalfas, N. Tomczyk, K. Giles, M. Morris, K. Thalassinos, Anal. Chem. 2019, 91, 75547561.

[24] K. Giles, J. Ujma, J. Wildgoose, S. Pringle, K. Richardson, D. Langridge, M. Green, Anal. Chem. 2019, 91, 8564-8573.

[25] P. Westermark, A. Andersson, G. T. Westermark, Physiol. Rev. 2011, 91, 795-826.

[26] A. Abedini, A. M. Schmidt, FEBS Lett. 2013, 587, 1119-1127.

[27] G. Ryan, L. Jobe, T. Briscoe, Drug Des. Devel. Ther. 2008, 203.

[28] F. W. McLafferty, Z. Guan, U. Haupts, T. D. Wood, N. L. Kelleher, J. Am. Chem. Soc. 1998, 120, 4732-4740.

[29] K. Breuker, F. W. McLafferty, Angew. Chemie - Int. Ed. 2003, 42, 4900-4904.

[30] E. R. Badman, S. Myung, D. E. Clemmer, J. Am. Soc. Mass Spectrom. 2005, 16, 1493-1497.

[31] Y. Xu, L. Mayne, S. W. Englander, Nat. Struct. Biol. 1998, 5, 774-778.

[32] Z. Ridgway, C. F. S. Eldrid, A. Zhyvoloup, A. Ben-Younis, D. Noh, K. Thalassinos, D. P. Raleigh, Biochemistry 2020, acs.biochem.9b01109.

[33] P. Marek, A. M. Woys, K. Sutton, M. T. Zanni, D. P. Raleigh, Org. Lett. 2010, 12, 4848-4851.

[34] G. N. Sivalingam, A. Cryar, M. A. Williams, B. Gooptu, K. Thalassinos, Int. J. Mass Spectrom. 2018, 426, 29-37. 

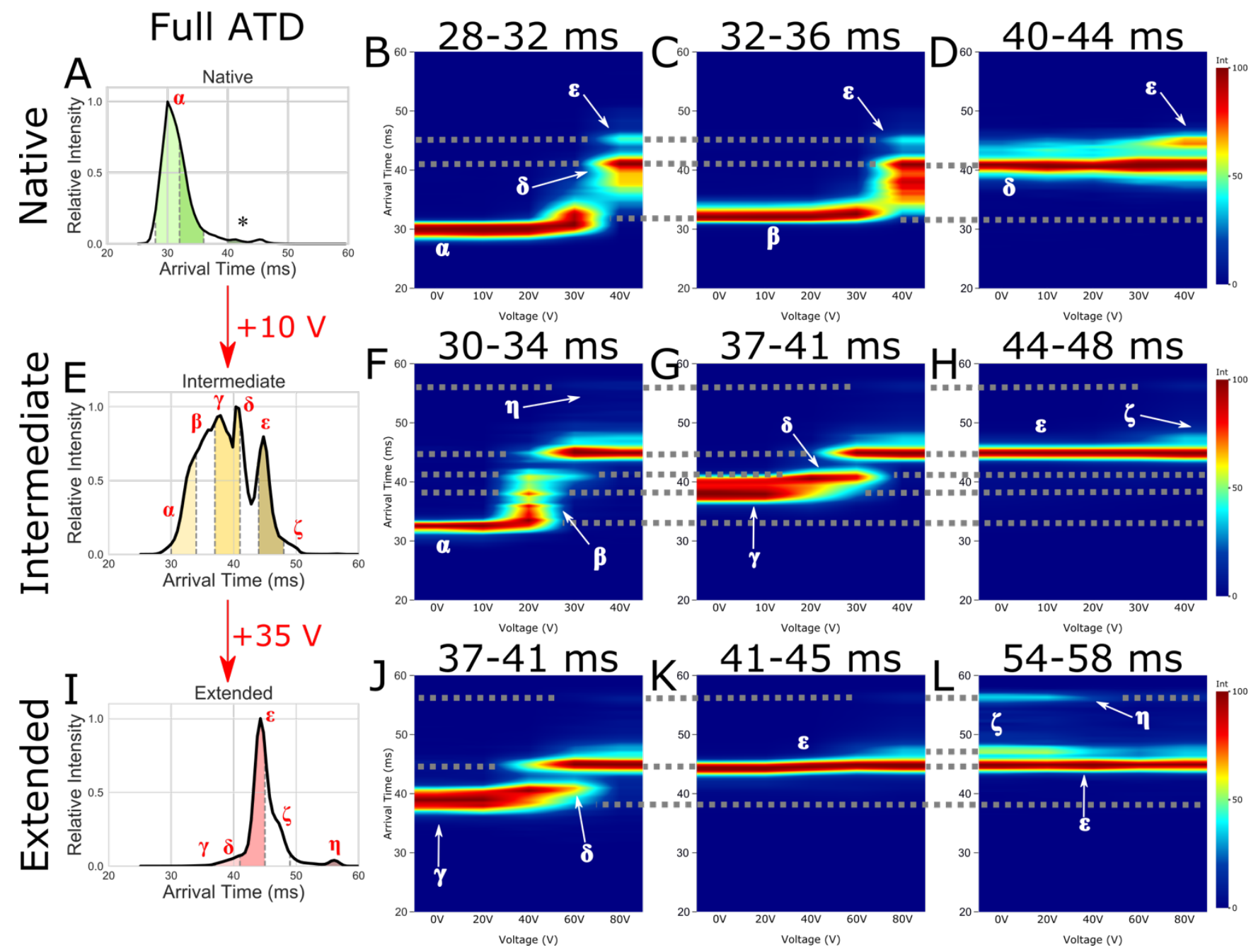

Figure 1 ATD for native (A), intermediate $(E)$ and extended $(I)$ CytC $(+7)$ with sub-population slices taken for CA denoted by dashed grey lines, filled with green (native), yellow (intermediate) and red (extended). Also shown are CA fingerprints for sub-populations of interest corresponding to native (B, $F, J)$, intermediate (C, $G, K)$ and extended $(D, H, L)$ states, with interpopulation conformations joined by dotted grey lines. Identified conformations of interest are labelled as $\alpha, \beta, y, \delta, \varepsilon$, $\zeta$ and $\eta$. The * denotes the low intensity species mobility selected in plot (D). 

$\mathrm{A} \quad \alpha \longrightarrow \beta \longrightarrow \delta$
$\mathrm{B} \quad \alpha \longrightarrow \beta \longrightarrow \gamma \longrightarrow \delta \longrightarrow \delta \longrightarrow{ }_{\eta}^{\zeta}$

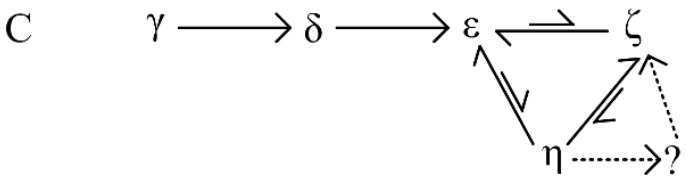

Figure 2 Transitional pathways of CytC unfolding. 

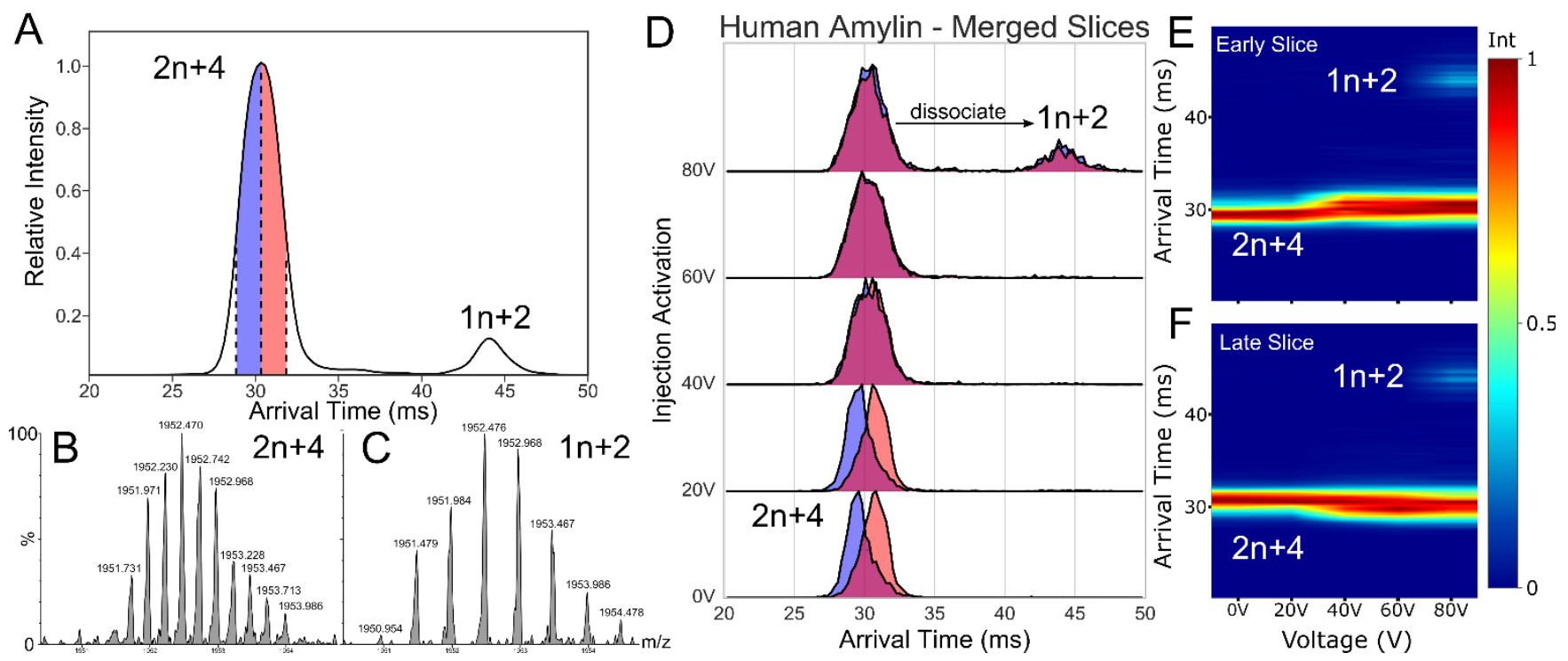

Figure 3 Slice-CA of hIAPP A) ATD of $1952 \mathrm{~m} / \mathrm{z}$ species showing a mix of monomer $(1 n+2)$ and dimer (2n+4) B) isotopic peak envelope for dimer C) isotopic peak envelope for monomer D) stacked IM plots of early slice (blue) and late slice (red) E) CA fingerprint of early slice F) CA fingerprint of late slice 